\title{
Patient, Family, and Center-Based Factors Associated with Attrition in Neonatal Clinical Research: A Prospective Study
}

\author{
Sara B. DeMauro ${ }^{a, b}$ Scarlett L. Bellamy ${ }^{b, c}$ Melissa Fernando ${ }^{b}$ Julie Hoffmann ${ }^{d}$ \\ Teresa Gratton ${ }^{\mathrm{e}}$ Barbara Schmidt ${ }^{\mathrm{a}}$ b PROP Investigators \\ ${ }^{a}$ Division of Neonatology, The Children's Hospital of Philadelphia, Philadelphia, PA, USA; ${ }^{b}$ University of \\ Pennsylvania Perelman School of Medicine, Philadelphia, PA, USA; ' Dornsife School of Public Health, Drexel

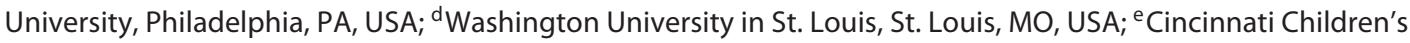 \\ Hospital Medical Center, Cincinnati, OH, USA
}

\section{Keywords}

Attrition · Clinical research $\cdot$ Prematurity $\cdot$ Follow-up

\begin{abstract}
Background: Attrition, or loss to follow-up, presents a significant threat to the integrity and validity of longitudinal clinical research. Little is known about predictors of attrition in neonatal clinical research, and no prior studies have examined how families' experiences participating in research with their infants influences study compliance. Objective: To identify novel factors that were associated with attrition over 1 year of study follow-up among preterm infants enrolled in the multicenter Prematurity and Respiratory Outcomes Program (PROP) observational study. Methods: At discharge, research coordinators estimated the likelihood of attrition. The parents completed questionnaires about their experience with the study at discharge and at 1 year corrected age. The primary endpoint was completion of 4 PROP interviews during the first year. Logistic models were used to evaluate the associations between infant, family, and center-based characteristics and attrition. Results: Among 318 children, 283 (89\%) met the primary endpoint. In bivariate analyses, lower maternal education, more peo-
\end{abstract}

\section{KARGER}

(c) 2019 S. Karger AG, Basel

E-Mail karger@karger.com

www.karger.com/neo ple in the household, public insurance, and site were associated with attrition $(p<0.05)$. Parent survey responses, infant characteristics, and site characteristics were unrelated to attrition. Coordinators' prediction of attrition was associated with completion of early study interviews; this effect waned over time. In multivariable analyses, lower maternal education and more people in the household were the factors most strongly associated with attrition. Conclusion: Future neonatal research should evaluate novel strategies to decrease the burden associated with study participation and reinforcement of study goals with families who have lower educational levels to facilitate participation and decrease attrition bias.

(c) 2019 S. Karger AG, Basel

\section{Introduction}

Attrition, or loss to follow-up, threatens the integrity and validity of longitudinal clinical research [1]. Only 5\% of studies achieve 100\% follow-up [2]. The direction of ascertainment bias caused by attrition is uncertain and unpredictable. In a systematic review of neonatal studies that reported 18 - to 24 -month corrected age (CA) out- 
comes, there was a nearly $1 \%$ increase in developmental impairment for every $1 \%$ decrease in the follow-up rate [2]. Thus, children who were lost were more likely to be unimpaired. Similar studies have predicted that children not evaluated at 2 years would have better cognitive outcomes than those who were seen; however, these predictions cannot be confirmed [3]. In contrast, in three regional cohorts and several small studies, preterm-born children who were difficult to follow up (but were eventually seen) and dropouts from research studies or clinical care were more likely to be impaired than those followed up with ease [4-9]. Given these conflicting results, efforts to minimize ascertainment bias by reducing attrition are essential.

Prior studies have not consistently identified the same demographic or socioeconomic predictors of attrition in neonatal clinical research, and no prior studies have examined the associations between families' research participation experiences and study compliance [4-7]. To better understand factors associated with attrition in neonatal clinical research, we performed an ancillary study to the multicenter Prematurity and Respiratory Outcomes Program (PROP) [8]. The objective was to identify novel factors measured at the time of hospital discharge that were associated with loss to follow-up at 12 months CA.

\section{Methods}

PROP was a prospective multicenter observational study that enrolled infants at gestational ages $<29$ weeks at $\leq 7$ days of life [8-10]. Exclusion criteria were: significant congenital heart disease; upper airway, lung, or chest wall anomalies; significant congenital malformations or syndromes; uncertain viability; or family unlikely to be available for long-term follow-up. Eight hundred thirty-five of 1,597 eligible children were enrolled (52.3\%). The reasons families were not approached or consent was not obtained are detailed elsewhere; importantly, the families of 123 infants were considered unlikely to be available for follow-up and the parents of 128 infants were unavailable for consent conversations [8]. PROP collected extensive in-hospital data, parent questionnaires when the children were $3,6,9$, and 12 months CA, and infant assessments at 1 year CA. The primary outcome was a measure of respiratory morbidity over the first year of life, incorporating the 4 parent questionnaires [10]. The PROP clinical centers were academic sites with experience conducting neonatal clinical research and funded by the NHLBI as part of a consortium of single-center and multicenter projects [8].

The PROP Attrition Reduction (PROP-AR) Study was performed as an ancillary to the primary study at 10 PROP sites. The enrolled infants survived until first discharge home. Only firstborn twins or higher-order multiples were enrolled, and children born to parents who did not speak English or Spanish as a primary language were excluded.

\section{Study Measures}

Before the PROP-AR Study began, research coordinators at each site were interviewed about their prior experience in research, interactions with families and clinical staff, the institutional clinical research infrastructure, and plans to limit attrition in PROP (see online Supplemental Information and Appendix; for all online suppl. material, see www.karger.com/doi/10.1159/000494105). The interviews were recorded with the permission of the coordinators.

During the week before each participant's anticipated hospital discharge, the research coordinator completed an online survey that asked about the coordinator's estimation of the likelihood that the family would continue to participate in PROP at 3, 6, 9, and 12 months CA. For example, "What is the probability that this PROP participant will complete the 3 month questionnaire? (Please provide a number between 0 (I don't think this family will complete the 3 month questionnaire) to 100 (I'm sure this family will follow up at 3 months)." The coordinators were instructed to answer these questions based on their subjective experiences with the participants' families during neonatal hospitalization.

The research coordinator then distributed a confidential questionnaire to each participant's parent or guardian (Fig. 1). The questionnaires were returned to the PROP Data Coordinating Center at the University of Pennsylvania in pre-addressed and stamped envelopes. The 10 Likert-style questions in the questionnaire were adapted from 2 valid and reliable surveys: the Critical Care Family Satisfaction Survey (CCFSS) and the Family Satisfaction with Care in the Intensive Care Unit (FS-ICU24) survey [11, 12]. The same questionnaire was administered at a clinic visit or mailed to the home with a self-addressed and stamped return envelope at 12 months CA.

\section{Primary Outcome, Analyses, and Sample Size}

The primary outcome of the PROP-AR Study was completion of all 4 post-discharge questionnaires. Factors potentially predictive of attrition (failure to complete all 4 questionnaires) were divided into 3 categories: (1) coordinator or site based, (2) parent based, and (3) infant based. We performed standard bivariate analyses to compare these characteristics between the group of children for whom all 4 questionnaires were completed ("complete") and the group of children for whom fewer than 4 questionnaires were completed ("incomplete"). Finally, we used a multivariable logistic regression model to determine which factors were most strongly associated with attrition. Site and all factors that were different between the groups at the $p<0.2$ level in bivariate testing were included in a full model with "complete participation" as the dependent variable. Then, insignificant factors were removed by backward elimination until only site and statistically significant factors remained in the final reduced model.

The percentage of children with complete participation at each site was used as a site-based measure of attrition. Relationships between this continuous measure and other site characteristics (e.g., the number of coordinators who worked on the study) were evaluated with standard bivariate tests.

We planned to enroll at least 275 participants in this hypothesis-generating observational study. The analyses were performed with Stata IC/13.0 (StataCorp, College Station, TX, USA), and $p<$ 0.05 was considered statistically significant. 
1. I am the infant's:

Mother Guardian

Father Other

2. Before your baby was born, were you ever involved as a family member of a patient in a neonatal intensive care unit (NICU)?

Yes No

3. Will the baby live with you in your home?

Yes No

4. Where do you live?

In the city where the hospital is located Out of town

5. Do you know at least one member of the PROP research team?

Yes No

Please circle one of the five answers that best describes how satisfied you were with the care that the research team provided for you and your child while in the NICU (options: Very Satisfied, Satisfied, Unsure, Not satisfied, Very Dissatisfied).

1. The research team was available to speak with me on a regular basis

2. The research team provided clear explanation of tests, procedures, and treatment

3. The research team provided clear answers to my questions

4. The research team was supportive and encouraging during my infant's stay in the NICU

5. The research team treated my baby with courtesy, respect, and compassion

6. The research team treated me with courtesy, respect, and compassion

7. The research team and the medical team (doctors and nurses) communicated with each other

8. The research team prepared me for the aspects of the study that will be completed after discharge

9. The research team protected my privacy and my baby's privacy

10. Overall, we had a positive experience participating in PROP

Is there anything in particular that made your experience participating in PROP difficult or challenging?

Is there anything in particular that made your experience participating in PROP worthwhile or rewarding?

Fig. 1. PROP-AR Study discharge and 12-month parent questionnaire.

\section{Results}

Between July 2012 and June 2014, 330 participants were enrolled in the PROP-AR Study. Of these, 12 were excluded ( 5 were placed in protective custody, 5 died after enrollment, and 2 withdrew from PROP), leaving 318 in the analysis cohort. In this cohort, participation in the PROP follow-up telephone questionnaires was: 304 at 3 months (95.6\%), 306 at 6 months (96.2\%), 300 at 9 months (94.3\%), and 300 at 12 months CA (94.3\%). Of those who completed the 12-month questionnaire, 149 (49.7\%) did the questionnaire by phone, 148 (49.3\%) participated in an in-person visit, and $3(1 \%)$ remained in hospital at 12 months. In general, sites that performed infant pulmonary function testing at 12 months completed the 12-month questionnaire in person. All 4 questionnaires were completed for
283 participants $(89.0 \%)$. The baseline medical and sociodemographic characteristics of the children with complete study participation, as compared to those with incomplete participation, are displayed in Table 1. Incomplete participation was significantly associated with lower maternal education, more people living in the household, and public insurance. In the bivariate analyses, attrition was significantly associated with site $(p=0.012)$.

There were no differences between the groups in the frequency of common inpatient morbidities (surgical necrotizing enterocolitis, ligation of the ductus arteriosus, culture-proven sepsis, or retinopathy of prematurity). Similarly, post-discharge use of medical technologies (continuous positive airway pressure, oxygen, ventilator, tracheostomy, feeding tubes, and home monitors) was not associated with complete study participation. 
Table 1. Characteristics of the study participants and their families

\begin{tabular}{|c|c|c|c|c|}
\hline & $\begin{array}{l}\text { All study } \\
\text { participants } \\
(n=318)\end{array}$ & $\begin{array}{l}\text { Incomplete study } \\
\text { participation } \\
(n=35)\end{array}$ & $\begin{array}{l}\text { Complete study } \\
\text { participation } \\
(n=283)\end{array}$ & $p$ value \\
\hline Birth weight, g & $909 \pm 221$ & $928 \pm 30$ & $906 \pm 226$ & 0.58 \\
\hline Gestational age, weeks & $26.3 \pm 1.4$ & $26.5 \pm 1.5$ & $26.2 \pm 1.4$ & 0.22 \\
\hline Multiple gestation & $50(15.7)$ & $3(8.6)$ & $47(16.6)$ & 0.32 \\
\hline Maternal race & & & & 0.32 \\
\hline Black & $122(38.4)$ & $17(48.6)$ & $105(37.1)$ & \\
\hline White & $190(59.8)$ & $18(51.4)$ & $172(60.8)$ & \\
\hline Other & $6(1.9)$ & $0(0)$ & $6(2.1)$ & \\
\hline Mother's highest level of education completed & & & & 0.024 \\
\hline$<12$ th grade & $57(19.6)$ & $11(36.7)$ & $46(17.6)$ & \\
\hline Number of people who live in the household (including baby) & & & & 0.010 \\
\hline 2-3 people & $116(36.5)$ & $12(34.3)$ & $104(36.8)$ & \\
\hline $4-6$ people & $173(54.4)$ & $15(42.9)$ & $158(55.8)$ & \\
\hline$\geq 7$ people & $29(9.1)$ & $8(22.9)$ & $21(7.5)$ & \\
\hline Private insurance & $86 / 316(27.2)$ & $3 / 35(8.6)$ & $83 / 281(29.5)$ & 0.009 \\
\hline \multicolumn{5}{|c|}{ Among the families who completed the PROP-AR discharge questionnaire $\mathrm{a}$} \\
\hline Family reports prior experience with NICU & 28/197 (14.2) & 2/17 (11.8) & $26 / 180(14.4)$ & 0.76 \\
\hline $\begin{array}{l}\text { Family reports living "out of town" or not in the city where } \\
\text { the hospital is located }\end{array}$ & 93/194 (47.9) & $10 / 17(58.8)$ & $83 / 177$ (46.9) & 0.35 \\
\hline $\begin{array}{l}\text { Family reports being familiar with at least one member of } \\
\text { the PROP research team }\end{array}$ & $152 / 193(78.8)$ & $13 / 17(76.5)$ & $139 / 176(79.0)$ & 0.81 \\
\hline
\end{tabular}

Results are presented as $n(\%)$ or mean \pm standard deviation. Italics denote significance. ${ }^{\text {a }}$ Results are for families who answered each question on the PROP-AR discharge questionnaire.

In general, the coordinators correctly predicted decreasing compliance over time (Table 2). On an individual-subject basis, the unadjusted odds that the coordinator's prediction was correct also appeared to decrease over time. Predicted compliance was highly site dependent $(p<0.001)$. Sites with high mean predicted compliance also tended to have high rates of study completion $(r=0.65, p=0.04)$. All sites provided modest financial incentives to families to participate in calls or study visits. These were generally discussed during the consent process, and most coordinators did not think that this practice influenced consent rates or study participation.

A mean of 6.7 (range 3-13) research coordinators were employed at each site over the duration of the PROP study. The total number of study coordinators at each site and the duration of coordinator participation were both unrelated to attrition. Factors related to the research cul- ture at each institution, as reported by the coordinators during the pre-study interviews, were unrelated to attrition.

The parents completed and returned 64\% $(n=204 / 318)$ of the PROP-AR Study discharge questionnaires (site range $38-100 \%) ; 91 \%$ of the families who returned the discharge questionnaire and $85 \%$ of the families who did not return the discharge questionnaire had complete participation in the main PROP study $(p=0.1)$. Children for whom the discharge questionnaire was not returned were more likely to be from a single-parent household (34 vs. $20 \%, p=0.005$ ) and were more likely to have mothers who described themselves as black or African American (49vs. $32 \%, p=0.026)$. Other characteristics were similar between the group with and the group without a PROP-AR Study parent discharge questionnaire. 
Table 2. Research coordinator predictions of participation in the PROP study over time

\begin{tabular}{lllll}
\hline Visit & $\begin{array}{l}\text { Mean } \\
\text { number } \pm \text { SD }\end{array}$ & $\begin{array}{l}\text { Median } \\
\text { number (IQR) }\end{array}$ & $\begin{array}{l}\text { OR for compliance } \\
(95 \% \text { vI })\end{array}$ \\
\hline 3 months & $87 \pm 16$ & $95(85-95)$ & $1.04(1.02-1.06)$ & 0.001 \\
6 months & $85 \pm 18$ & $90(80-95)$ & $1.03(1.01-1.05)$ & 0.009 \\
9 months & $86 \pm 17$ & $90(80-95)$ & $1.02(1.00-1.04)$ & 0.049 \\
12 months & $84 \pm 19$ & $90(75-95)$ & $1.01(0.99-1.04)$ & 0.11 \\
\hline
\end{tabular}

The mean response on the 10 Likert-style parent discharge questions was $4.7 \pm 0.5$. Thus, parents tended to report a strongly positive experience participating in PROP with their child. The parents were provided with the same questionnaire at 12 months CA, and 191 (60\%) of the questionnaires were returned. The mean response was $4.9 \pm 0.2$, or "very satisfied." There was no relationship between parent responses on these two questionnaires and attrition.

In the multivariable logistic model, infant characteristics, parental perspectives on study participation, and site were not significantly associated with attrition. Maternal education below a high school degree (adjusted OR 0.38, 95\% CI 0.15-0.94) and living with more than 7 people in the household (adjusted OR 0.54, 95\% CI 0.30-0.98) were the factors most strongly associated with attrition.

\section{Discussion}

In this study, we explored infant, parent, and sitebased factors to ascertain whether these were related to attrition in a longitudinal research study. A lower level of maternal education and a higher number of people in the home were the factors most strongly and independently associated with attrition. We suggest that these factors are surrogate measures of socioeconomic status. We were unable to identify site characteristics or parental perspectives on the experience of research participation that might also predict attrition. Though the research coordinators reported widely different approaches to maintaining contact with families, staffing strategies, and research cultures and there was substantial site variation in study staffing, these characteristics were not associated with study participation.

Attrition has been studied in several selected adult populations and linked with patient characteristics such as age, cognitive status, and smoking [13-15]. In three randomized trials of behavioral weight loss strategies, attrition increased with lower educational attainment, higher body mass index, lack of insurance, and younger age [16]. In a small study of pediatric burn patients, the children who did not take part were significantly different from those that participated, and dropouts were more likely to have a mental health diagnosis [17].

There is little known about factors that are predictive of attrition or strategies to prevent attrition in neonatology. Because research participants enrolled in the newborn period are dependent on caregivers throughout the follow-up period for most studies and clinical programs, predictors of attrition may be related to the participant, the family, or relationships between the two. For example, some have suggested that parents who are unprepared to cope with a child's illness are less likely to comply with follow-up [18]. A few studies have suggested that lower gestational age, center, female gender, lower maternal educational attainment, maternal race, and lack of an intact family are associated with attrition in pediatric and neonatal care $[4-7,19]$. In a 5-center North American trial, withdrawal or loss to follow-up at 36 months was associated with gestational age, center, and socioeconomic/social support [4]. A 1995 German study reported that children who were multiples were more likely to follow up, while girls and children of mothers with lower educational attainment were more likely to drop out [5]. Similarly, regional Australian studies have reported that children who were difficult to follow up at 5 and 8 years were more likely to come from disrupted families and had mothers with less education $[7,9]$. In the current study, neither inhospital nor post-discharge medical characteristics of the children were related to attrition, but sociodemographic factors were strongly related to study completion. Importantly, sociodemographic factors are also strongly related to both high-risk infants' developmental outcomes as well as developmental trajectories [20-23]. Thus, attrition in studies such as PROP may be nonrandom, and attrition among children with differing sociodemographic backgrounds is likely to bias the results of longitudinal studies [24].

Others have reported that clinical center is one of the factors most strongly predictive of participant loss in longitudinal research [4]. While site was highly significant in bivariate analyses, it was not significant in our multivariate analyses. Despite frequent interaction with site research coordinators, we were unable to explain or reduce the significant variation in rates of PROP-AR Study survey completion across sites. We attempted to elucidate which aspects of the local research culture were associ- 
ated with retention of participants; unfortunately, none of the factors examined were predictive of site-specific attrition rates.

A 2007 systematic review identified multiple narrative reports but no quantitative evaluations of different strategies to prevent attrition in adult populations [25]. This study suggested that successful retention strategies can be categorized into three themes: (1) respect for patients, including their ideas and their time commitment to the research; (2) details related to patient tracking, including procedures for subjects lost to follow-up; and (3) support and training of study personnel [25]. The authors applied these strategies in the adult intensive care unit and reported 1- and 5-year follow-up rates of 91 and $86 \%$, respectively. No similar studies have been published in pediatrics or neonatology. The results of the adult studies cannot necessarily be generalized to children, who depend on parents for continued study participation.

The large cohort, detailed longitudinal follow-up, and multicenter nature of PROP made it ideal for evaluating factors related to successful retention of study participants. While frequent phone contact over 1 year does not represent the usual expectations for neonatal follow-up, many research protocols and clinical scenarios require continued family engagement after discharge and would benefit from targeted strategies to limit attrition. Frequent contact between the research team and families throughout hospitalization, prior to discharge, and after discharge may explain at least in part the low rate of attrition in PROP. This approach ought to be incorporated into future neonatal studies that require long-term follow-up.

Our study suggests that infants at high risk of loss to follow-up can be identified before hospital discharge. The relationship between attrition and sociodemographic risk factors suggests some potential solutions. Additional incentives (including nonfinancial incentives), strategies to make research participation less burdensome, and reinforcement of the goals of the study with families who have lower educational levels may facilitate participation. Important next steps in this work will be to develop and test strategies to limit attrition among study participants at high risk of loss to follow-up. Such strategies are critical, because differential loss to follow-up may lead to misleading conclusions in research studies.

The low attrition in PROP may have limited the power of the study to detect predictors of attrition. The response rates on the parent questionnaires may have limited our ability to understand the experience of all families with the research study. On the other hand, there was no significant difference in attrition between families who completed the PROP-AR Study questionnaires and those who did not. Lastly, we do not have any data about whether the coordinator who assessed the likelihood of compliance for each family was the same coordinator who obtained the original study consent or the duration of that coordinator's relationship with the family.

Research coordinators are the key to a successful study team. They build rapport, facilitate communication, and ensure that families enjoy research participation. In this study, the coordinators identified families at risk of attrition over at least the first 9 months after discharge. Therefore, sophisticated parent questionnaires and data collection processes may not be necessary. It may instead be more important for the team to have a dedicated research coordinator who understands the babies and their families. This, paired with a thoughtful and focused approach to maintaining engagement with and supporting children and families who have risk factors for attrition, may be the optimal strategy for limiting attrition. Further research will be essential for developing targeted, evidencebased strategies to reduce attrition bias in neonatal clinical research.

\section{Acknowledgments}

This study was supported by PROP grants U01 HL101794, U01 HL101456, U01 HL101798, U01 HL101813, U01 HL101465, U01 HL101800, and R01 HL105702. We gratefully acknowledge all PROP participants and their families, as well as the PROP staff at the University of Pennsylvania Data Coordinating Center and the Research Coordinators at the PROP clinical sites.

\section{Statement of Ethics}

The PROP-AR Study was approved as a modification to the main PROP protocol by the Institutional Review Boards at the University of Pennsylvania and all participating clinical centers; additional consent was not required.

\section{Disclosure Statement}

The authors have no conflicts of interest relevant to this article to disclose. 


\section{References}

1 Marcellus L. Are we missing anything? Pursuing research on attrition. Can J Nurs Res. 2004 Sep;36(3):82-98.

2 Guillén U, DeMauro S, Ma L, Zupancic J, Roberts R, Schmidt B, et al. Relationship between attrition and neurodevelopmental impairment rates in extremely preterm infants at 18 to 24 months: a systematic review. Arch Pediatr Adolesc Med. 2012 Feb;166(2):17884.

3 Castro L, Yolton K, Haberman B, Roberto N, Hansen NI, Ambalavanan N, et al. Bias in reported neurodevelopmental outcomes among extremely low birth weight survivors. Pediatrics. 2004 Aug;114(2):404-10.

4 Aylward GP, Hatcher RP, Stripp B, Gustafson NF, Leavitt LA. Who goes and who stays: subject loss in a multicenter, longitudinal followup study. J Dev Behav Pediatr. 1985 Feb;6(1) 3-8.

5 Wolke D, Söhne B, Ohrt B, Riegel K. Followup of preterm children: important to document dropouts. Lancet. 1995 Feb;345(8947): 447.

6 Callanan C, Doyle L, Rickards A, Kelly E, Ford G, Davis N. Children followed with difficulty: how do they differ? J Paediatr Child Health. 2001 Apr;37(2):152-6.

7 Constantine WL, Haynes CW, Spiker D, Kendall-Tackett K, Constantine NA. Recruitment and retention in a clinical trial for low birth weight, premature infants. J Dev Behav Pediatr. 1993 Feb;14(1):1-7.

8 Pryhuber GS, Maitre NL, Ballard RA, Cifelli D, Davis SD, Ellenberg JH, et al.; Prematurity and Respiratory Outcomes Program Investigators. Prematurity and Respiratory Outcomes Program (PROP): study protocol of a prospective multicenter study of respiratory outcomes of preterm infants in the United States. BMC Pediatr. 2015 Apr;15(1):37.
9 Poindexter BB, Feng R, Schmidt B, Aschner JL, Ballard RA, Hamvas A, et al.; Prematurity and Respiratory Outcomes Program. Comparisons and limitations of current definitions of bronchopulmonary dysplasia for the Prematurity and Respiratory Outcomes Program. Ann Am Thorac Soc. 2015 Dec;12(12): 1822-30.

10 Keller RL, Feng R, DeMauro SB, Ferkol T, Hardie W, Rogers EE, et al.; Prematurity and Respiratory Outcomes Program. Bronchopulmonary dysplasia and perinatal characteristics predict 1-year respiratory outcomes in newborns born at extremely low gestational age: a prospective cohort study. J Pediatr. 2017 Aug;187:89-97.e3.

11 Wall RJ, Engelberg RA, Downey L, Heyland DK, Curtis JR. Refinement, scoring, and validation of the Family Satisfaction in the Intensive Care Unit (FS-ICU) survey. Crit Care Med. 2007 Jan;35(1):271-9.

12 Karlsson C, Tisell A, Engström A, Andershed B. Family members' satisfaction with critical care: a pilot study. Nurs Crit Care. 2011 JanFeb;16(1):11-8.

13 Young AF, Powers JR, Bell SL. Attrition in longitudinal studies: who do you lose? Aust N Z J Public Health. 2006 Aug;30(4):353-61.

14 Chatfield MD, Brayne CE, Matthews FE. A systematic literature review of attrition between waves in longitudinal studies in the elderly shows a consistent pattern of dropout between differing studies. J Clin Epidemiol. 2005 Jan;58(1):13-9.

15 Gades NM, Jacobson DJ, McGree ME, Lieber MM, Roberts RO, Girman CJ, et al. Dropout in a longitudinal, cohort study of urologic disease in community men. BMC Med Res Methodol. 2006 Dec;6(1):58

16 Goode RW, Ye L, Sereika SM, Zheng Y, Mattos M, Acharya SD, et al. Socio-demographic, anthropometric, and psychosocial predictors of attrition across behavioral weight-loss trials. Eat Behav. 2016 Jan;20:27-33.
17 McQuaid D, Barton J, Campbell E. Researchers BEWARE! attrition and nonparticipation at large. J Burn Care Rehabil. 2003 Jul-Aug; 24(4):203-7.

18 Wariyar UK, Richmond S. Morbidity and preterm delivery: importance of $100 \%$ followup. Lancet. 1989 Feb;1(8634):387-8.

19 Cooper NA, Lynch MA. Lost to follow up: a study of nonattendance at a general paediatric outpatient clinic. Arch Dis Child. 1979 Oct 54(10):765-9.

20 Manley BJ, Roberts RS, Doyle LW, Schmidt B, Anderson PJ, Barrington KJ, et al. Social variables predict gains in cognitive scores across the preschool years in children with birth weights 500 to 1250 grams. J Pediatr. 2015 Apr;166(4):870-6.e1-2.

21 Hackman DA, Farah MJ. Socioeconomic status and the developing brain. Trends Cogn Sci. 2009 Feb;13(2):65-73.

22 Tong S, Baghurst P, Vimpani G, McMichael A. Socioeconomic position, maternal IQ, home environment, and cognitive development. J Pediatr. 2007 Sep;151(3):284-8, 288. e1.

23 Kilbride HW, Thorstad K, Daily DK. Preschool outcome of less than 801-gram preterm infants compared with full-term siblings. Pediatrics. 2004 Apr;113(4):742-7.

24 Doyle LW, Anderson PJ, Burnett A, Callanan C, McDonald M, Hayes M, et al.; Victorian Infant Collaborative Study (VICS) Group. Developmental disability at school age and difficulty obtaining follow-up data. Pediatrics. 2018 Feb;141(2):e20173102.

25 Tansey CM, Matté AL, Needham D, Herridge MS. Review of retention strategies in longitudinal studies and application to follow-up of ICU survivors. Intensive Care Med. 2007 Dec; 33(12):2051-7. 